\title{
The perceptual aspect of skilled performance in chess: Evidence from eye movements
}

\author{
NEIL CHARNESS \\ Florida State University, Tallahassee, Florida \\ and \\ EYAL M. REINGOLD, MARC POMPLUN, and DAVE M. STAMPE \\ University of Toronto, Toronto, Ontario, Canada
}

\begin{abstract}
Expert and intermediate chess players attempted to choose the best move in five chess positions while their eye movements were monitored. Experts were faster and more accurate than intermediates in choosing the best move. Experts made fewer fixations per trial and greater amplitude saccades than did intermediates, but there was no difference in fixation duration across skill groups. Examining the spatial distribution of the first five fixations for each position by skill group revealed that experts produced more fixations on empty squares than did intermediates. When fixating pieces, experts produced a greater proportion of fixations on relevant pieces than did intermediates. It is argued that expert chess players perceptually encode chess configurations, rather than individual pieces, and, consequently, parafoveal or peripheral processing guides their eye movements, producing a pattern of saccadic selectivity by piece saliency.
\end{abstract}

One of the most fascinating and impressive aspects of skilled performance is the ability of the experienced eye to encode at a glance the essence of briefly presented stimulus material, which is related to the domain of expertise (henceforth, domain-specific knowledge). For example, Kundel and Nodine (1975) showed expert radiologists $\mathrm{X}$-ray films for $200 \mathrm{msec}$. The experts were able to detect and name $70 \%$ of the abnormalities in the films.

Crucial to this process of rapid perception, particularly for visual displays that require multiple fixations for encoding, is the ability to encode large clusters of related information - that is, chunks - and to locate the most relevant areas, or identify the salient locations, on which to focus attention. In order to examine these early perceptual encoding processes, we required chess players at different skill levels to choose the best move for simple, tactically active chess positions while we monitored their eye-fixation patterns. If more skilled players can extract relational information about piece clusters more efficiently than less skilled players (e.g., in parallel, as seen in Reingold, Charness, Pomplun, \& Stampe, 2001; Reingold, Charness, Schultetus, \& Stampe, 2001), we hypothesize that skilled players' first few seconds of fixations will be characterized by a greater likelihood of fixating on empty squares (in order to maximize information extraction from

Preparation of this paper was supported by NIA Grant 5R01 AG13969 to N.C. and by a grant to E.R. from the Natural Science and Engineering Research Council of Canada. Correspondence should be addressed to N. Charness, Department of Psychology, Florida State University, Tallahassee, FL 32306-1270 (e-mail: charness@psy.fsu.edu). surrounding piece-occupied squares). Also, when more skilled players fixate squares occupied by pieces, they should be more likely to fixate salient pieces.

The most influential investigation of the perceptual aspects of skilled performance originated from the pioneering work on chess by de Groot (1946/1978) and Chase and Simon (1973a, 1973b). De Groot presented chess positions briefly $(2-15 \mathrm{sec})$ and then removed them from view. Even after such a brief exposure, the best chess players were able to reproduce the locations of the chess pieces almost perfectly (about $93 \%$ correct for positions containing about 25 pieces). More generally, performance in this task systematically varied as a function of skill. De Groot concluded that perception and memory were more important differentiators of chess expertise than was the ability to think ahead in the search for good moves. In a classic study, Chase and Simon (1973a, 1973b) replicated and extended de Groot's findings demonstrating that after viewing chess positions for $5 \mathrm{sec}$, chess masters were able to reproduce these positions much more accurately than less-skilled players. However, there was little difference as a function of expertise when random board configurations were used instead of game positions, indicating that the superior immediate memory performance of the skilled players was not attributable to the general superiority of their memory systems or processes (i.e., hardware aspects of memory). More recently, a very small but reliable advantage in recall for random configurations has been shown for expert players, although this is probably attributable to the occasional presence of familiar configurations in random positions (Gobet \& Simon, 1996a). 
Like de Groot (1946/1978), Chase and Simon (1973a, 1973b) hypothesized that much of the advantage of the skilled chess player lies in the early perceptual organization and internal representation of the chess position. The master is thought to use recognizable configurations of pieces, chunks and templates, as indexes to long-term memory structures that trigger the generation of plausible moves for use by a search mechanism. Search is thereby constrained to the more promising branches in the space of possible moves from a given chess position. The size of an expert's vocabulary of chess-related configurations was initially estimated to be 50,000-100,000 chunks (Simon \& Gilmartin, 1973), although small perceptual chunks are most likely supplemented by larger structures termed templates (Gobet \& Simon, 1996b, 1998), and the current estimate is around 300,000 chunks (Gobet $\&$ Simon, 2000). On the basis of earlier findings, Chase and Simon (1973a) concluded "that the most important processes underlying chess mastery are these immediate visual-perceptual processes rather than the subsequent logical-deductivethinking processes" (p. 215). Note that implied by the quote above is the distinction between an initial perceptual phase and a subsequent search phase of the problem-solving process (see also de Groot, 1946/1978; Simon \& Chase, 1973; Tikhomirov \& Poznyanskaya, 1966).

To investigate the perceptual phase of skilled performance may require the development of new research paradigms or the modification of existing ones. As Newell and Simon (1972) observed, although verbal think-aloud protocols provide a very rich source of information about the problem-solving process, it is necessary to supplement them with other sources in order to ensure that stable inferences are drawn. A potentially promising approach involves the measurement of eye movements to supplement reaction time, accuracy, and verbal report measures.

The present study illustrates the potential role of eyemovement measurement in supplementing traditional measures of performance, such as RT, accuracy, and verbal reports, as a means for investigating the perceptual aspect of skilled performance in general and chess skill in particular. Given the pivotal role played by eye-movement paradigms in the study of reading skill (see Rayner, 1998, for a review), it is surprising that there are very few empirical studies that have employed these techniques with chess (de Groot \& Gobet, 1996; Ellis, 1973; Jongman, 1968; Tikhomirov \& Poznyanskaya, 1966; Winikoff, 1967).

Reanalyzing the work of Jongman (1968), de Groot and Gobet (1996) reported no significant difference in the proportion of fixations on empty squares as a function of skill. These authors cautioned, however, that the negative results do not necessarily refute the chunking hypothesis. They pointed out that the crude frame-by-frame analysis of film records of eye movements and the transformation of gaze positions from a three-dimensional chessboard viewed by the players to a two-dimensional coordinate system may have resulted in the introduction of noise, making it difficult to estimate the accuracy of the computed gaze position. Furthermore, de Groot and Gobet demonstrated that skilled players made more fixations along the edges of squares (28.7\% of fixations), as compared with novices (13.7\%), providing some indication that the skilled players might be able to encode two or more pieces in a single fixation. They also noted a greater distance between successive fixations by skilled players, suggesting that they cover a larger area during a given fixation than do weaker players. In addition, de Groot and Gobet concluded on the basis of their analysis of retrospective verbal reports that the best players tended to perceive groups of pieces, rather than individual pieces.

Reynolds (1982) and Holding (1985) reexamined eyemovement data collected by Tikhomirov and Poznyanskaya (1966) and noted that many fixations did not fall on pieces but on empty squares. There was no report of systematic variation in the proportion of fixations on empty squares as a function of skill. However, the imprecision of the frame-by-frame analysis of film records that was used to extract eye-movement data makes it difficult to assess the reliability of these interpretations.

In recent studies, we have used eye-fixation data to supplement information available from reaction time data to demonstrate an early perceptual advantage through parallel encoding of chess relations for more skilled players in a simple check detection task (Reingold, Charness, Pomplun, \& Stampe, 2001) and to improve our understanding of the hazards of expertise (susceptibility to Stroop-like interference; Reingold, Charness, Schultetus, \& Stampe, 2001).

The check-detection task (Reingold, Charness, Pomplun, \& Stampe, 2001), in which a minimized $3 \times 3$ square chessboard was used, revealed that experts made fewer fixations per trial, and a greater proportion of those fixations were between pieces rather than on pieces, as compared with those of novices and intermediates. Such results are consistent with the hypothesis that highly skilled players have a larger visual span than do less-skilled ones. This hypothesis received additional support from a gazecontingent change blindness experiment showing that experts required a larger visual window than did novices to keep performance equivalent to that with unrestricted view.

In another check-detection task (Reingold, Charness, Schultetus, \& Stampe, 2001), a $5 \times 5$ section of the chessboard containing a King and two potential checking pieces (i.e., attackers) was employed. On some trials, one of two attackers was cued (colored red), and the task was to determine the checking status of the cued attacker while ignoring the other attacker. On other trials, the attackers were not cued. We documented that, unlike weaker players, experts did not benefit from the narrowing of the problem search space produced by cuing. In addition, expert players exhibited significant Stroop-like interference on trials in which a cued nonchecking attacker appeared together with an attacker that was checking (i.e., the checking status of the cued attacker was incongruent with the checking status of the configuration as a whole). These findings suggest automatic and parallel encoding processes for chess relations in experts. 
A major goal of the present study was to replicate and extend the previous findings to the more ecologically valid task of choosing the best move (henceforth, the movechoice task) with full chessboard displays. We chose the move-choice task for several reasons. First, eye-movement studies have long shown that the nature of the task can result in very different patterns of fixations for the same visual configuration (e.g., Yarbus, 1967). The fixation patterns for memorizing a chess position (de Groot \& Gobet, 1996) or for doing simple check detection (Reingold, Charness, Pomplun, \& Stampe, 2001) might not be representative of those in problem-solving situations. Second, de Groot (1946/1978) demonstrated that performance on the move-choice task (quality of move chosen) discriminates well between chess players at different levels of skill. We reasoned that focusing on the first 1-2 secs of eye fixations in each trial is important in order to attribute any potential skill differences to the perceptual, rather than to the problem-solving, phase. In other words, given that experts encode positions more quickly than intermediate players, going much beyond five fixations might lead to a skill-related confound of fixations in encoding the initial representation versus fixations that promote problem-solving processes (e.g., search through the space of possible moves). Hence, we investigated the spatial distribution of the first five fixations produced by players who attempted to choose the best move for a given position.

We tested two specific predictions. First, on the basis of the chunking hypothesis, we predicted that a greater proportion of fixations would occur on empty squares for experts, as compared with intermediates. Second, among fixations occurring on individual pieces, we predicted that a greater proportion of fixations would occur on salient pieces (i.e., tactically active pieces) for experts, as compared with intermediates. The latter prediction is based on a finding by de Groot and Gobet (1996) that the number and total duration of fixations landing on chess pieces during a memorization task were at least partially correlated with the degree of importance or relevance of these pieces in a given position and that the magnitude of this correlation increased as a function of skill. Similarly, on the basis of their simulation of eye-movement data collected by Tikhomirov and Poznyanskaya (1966), Simon and Barenfeld (1969) argued that fixations fell on what they defined as the salient pieces for the position. In the present study, we determined piece saliency for the five positions used in the experiment by asking two international masters to classify pieces as salient or as nonsalient. Figure 1 shows these positions with salient pieces surrounded by a bold frame.

\section{METHOD}

\section{Participants}

Twenty-four paid participants (12 intermediates, 12 experts), were included in this study. All participants had normal or corrected-to-normal vision. The Chess Federation of Canada (CFC) ratings for the expert players range from 2,100 to $2,350(M=2,238$, $S D=88.6)$. CFC ratings for the intermediates range from 1,400 to
$1,923(M=1,786, S D=142.5)$. The mean rating in the CFC is about 1,600 with a standard deviation of about 200. Players ranged in age between 18 and 34 . Informed consent was obtained, and the rights of the participants were protected.

\section{Materials}

The stimulus displays showed chessboards subtending a visual angle of $18.2^{\circ}$ horizontally and vertically and included chess pieces of approximately $1.9^{\circ}$ in diameter. The five experimental chess positions shown in Figure 1 were selected from Horowitz (1972) and Reinfeld (1945). The selected positions all had a clear best move and were tactically active. A position taken from Tikhomirov and Poznyanskaya (1966) was used for practice. For each experimental position, two international masters were asked to classify pieces as salient or as nonsalient. The percent agreement between the two judges in the classification of 102 pieces across all five positions was $98 \%$.

\section{Apparatus}

Eye movements were measured with an SR Research EyeLink system. After the system was calibrated, gaze-position error was less than $0.5^{\circ}$. The temporal resolution of the system was $4 \mathrm{msec}$. The on-line saccade detector of the eye tracker was set to detect saccades with an amplitude of $0.5^{\circ}$ or greater, with an acceleration threshold of $9,500^{\circ} / \mathrm{sec}^{2}$ and a velocity threshold of $30^{\circ} / \mathrm{sec}$.

\section{Procedure}

All players were presented with the practice position and then with the five experimental positions. Prior to every trial, instruction was given indicating who was to move (White or Black). The participants were then asked to fixate a marker in the center of the display. Following a buttonpress, a chessboard was presented. Players were asked to choose the best move as quickly and as accurately as possible. As soon as the participants made their selection, they ended the trial by pressing another button and naming the move. The experimenter monitored and recorded the accuracy of their performance.

\section{RESULTS}

For each player, the number of correct responses and median RT were computed across the five experimental trials. Experts were faster [experts, $M=11.7 \mathrm{sec}$; intermediates, $M=23.4 \mathrm{sec} ; t(22)=2.97, p<.01]$ and more accurate [experts, $M=4.75$ out of 5 ; intermediates, $M=$ 3.33 out of $5 ; t(22)=2.93, p<.01]$ than intermediates in choosing the best move. Experts also made about only half as many fixations per trial as intermediates [experts, $M=$ 44.3; intermediates, $M=83.6 ; t(22)=3.10, p<.01]$.

Figure 2 shows the distributions of saccade amplitude (Panel A) and fixation duration (Panel B). Consistent with the findings of de Groot and Gobet (1996), experts made larger amplitude saccades than did intermediates [experts, $M=4.02^{\circ}$; intermediates, $M=3.38^{\circ} ; t(22)=2.15, p<$ $.05]$. However, unlike de Groot and Gobet, who reported shorter fixation durations for skilled players as compared with weaker players, there was no significant difference across skill groups in the present study [experts, $M=253$; intermediates, $M=244 ; t(22)=0.56, p=.58]$. This discrepancy is likely due to the difference in the task performed by the players in the two studies. Whereas in the present study players were choosing the best move in a well-defined chess position, in the study by Jongman 

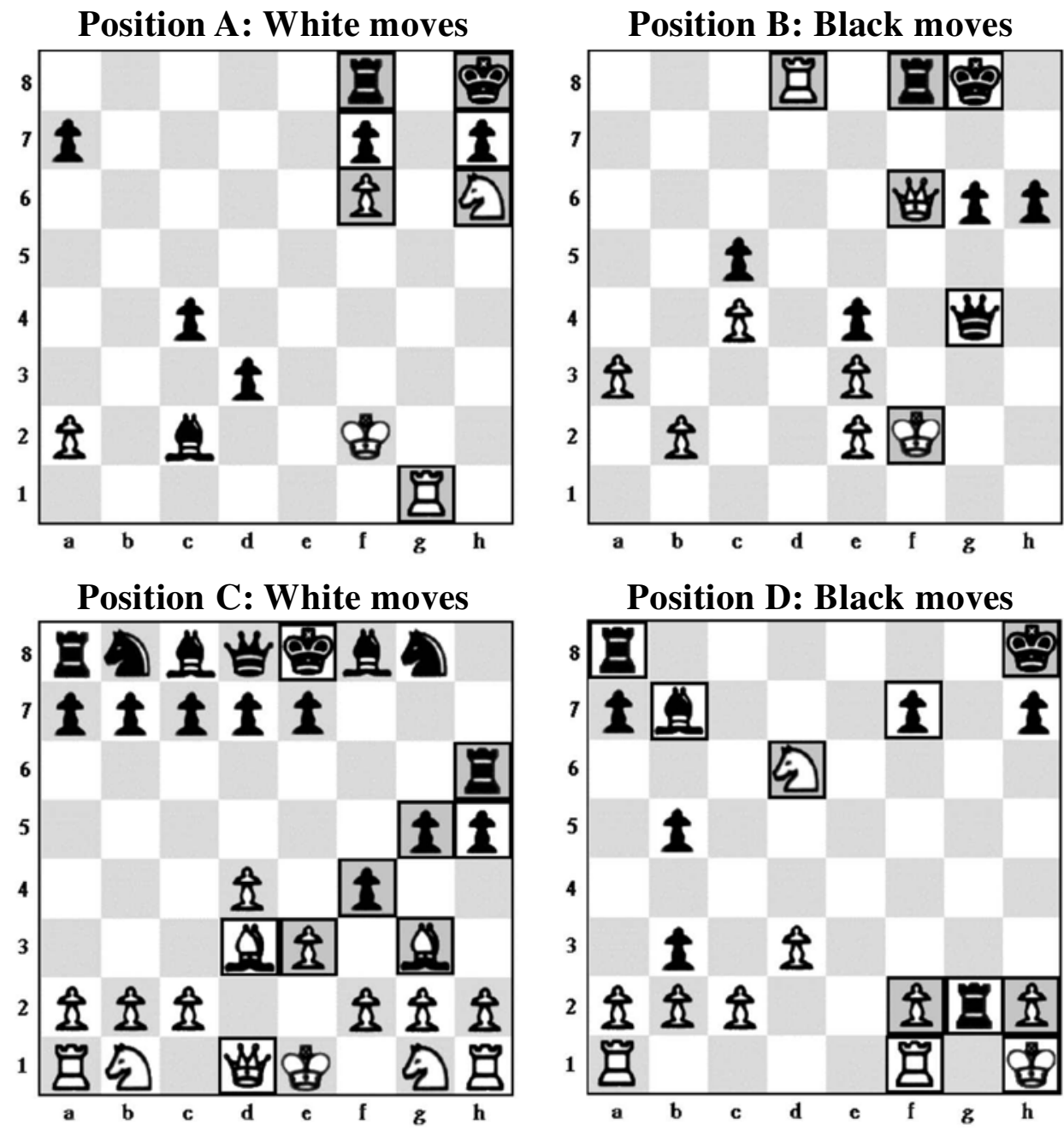

\section{Position E: White moves}

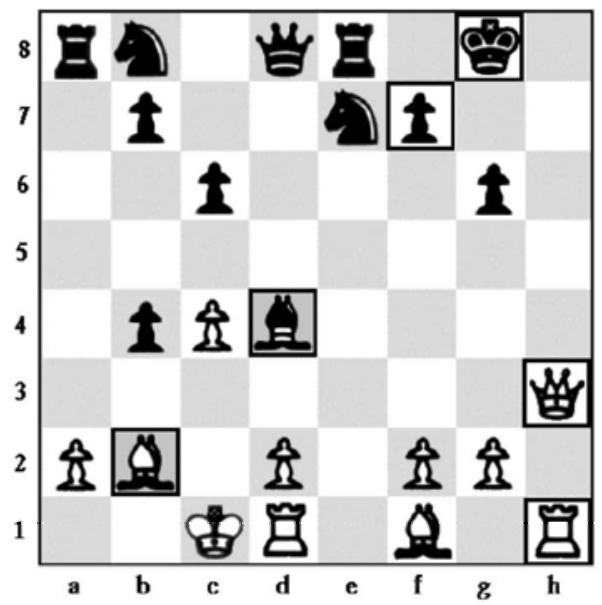

Figure 1. The five experimental positions used in the move-choice task. Chess pieces surrounded by a bold frame were judged to be salient in the position by two international masters. Position A: White Rook moves to g8 check, Black Rook takes White Rook, White Knight takes Pawn at f7 mate. Position B: Black Queen moves to h4 check, White King moves to escape, Black Queen takes White Queen. Position C: White Queen takes Pawn at h5 check, Black Rook takes White Queen, White Bishop moves to g6 mate. Position D: Black Rook moves to g1 check, White King takes Black Rook, Black Rook moves to g8 mate. Position E: White Queen moves to h8 check, Black Bishop takes White Queen, White Rook takes Black Bishop mate. 

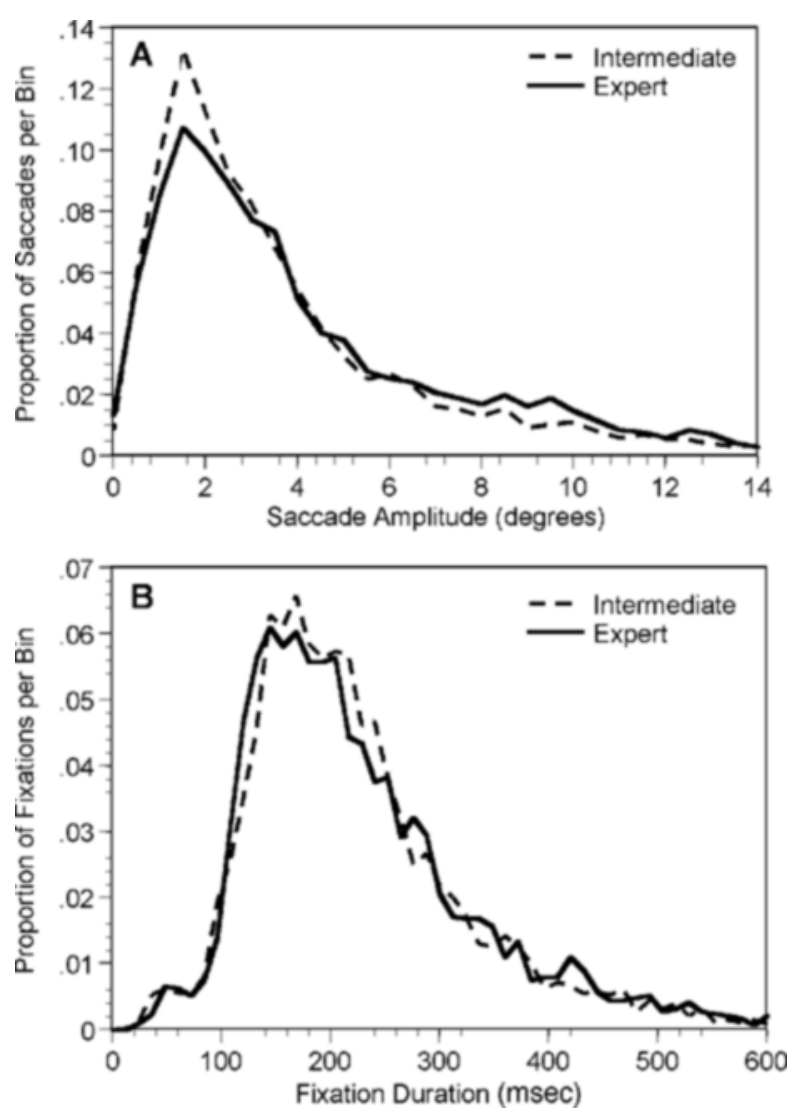

Figure 2. Histograms of saccade amplitude $($ Panel $\mathrm{A}$, bin size $=$ $0.5^{\circ}$ ) and fixation duration (Panel $B$, bin size $=12 \mathrm{msec}$ ) for $\mathrm{ex}$ perts and intermediates in the move-choice task.

(1968), analyzed by de Groot and Gobet, players were memorizing more ambiguous positions (players did not know whether it was White's or Black's move) in an immediate recall task. Further investigationinto the influence of task instructions and task type on skill differences in eye-movement patterns during visual tasks is needed. In some cases, experts make fewer, though same-duration, fixations (the check-detection task; Reingold, Charness, Pomplun, \& Stampe, 2001). In other cases, novices make longer fixations than do experts (e.g., in a driving task with dangerous situations; Chapman \& Underwood, 1998).

In order to investigate the spatial distribution of fixations produced by players during the early perceptual phase of attempting to chose the best move, the gaze positions of the first five fixations were recorded for each player and trial [average fixation duration: experts, $M=210$; intermediates, $M=199 ; t(22)=0.63, p=.54]$ and were classified as falling on an empty square or on a square occupied by a salient or a nonsalient piece. Figure 3 illustrates the skill differences in the spatial distribution of fixations for one of the experimental positions (Position A, see Figure 1). As can be clearly seen by comparing the scattergrams, consistent with the chunking hypothesis, experts produced a greater proportion of fixations on empty squares than intermediates [experts, $M=.52$; intermediates, $M=.41 ; t(22)=2.43, p<.05]$. In addition, consistent with de Groot and Gobet (1996), among fixations on pieces, experts produced a greater proportion of fixations on salient pieces than intermediates [experts, $M=$ .80 ; intermediates, $M=.64 ; t(22)=3.02, p<.01]$.

\section{DISCUSSION}

At the molar level, we observe faster and more accurate problem solving by experts in a move-choice task involving simple tactical positions. At the molecular level, over the first five fixations, we find more efficient encoding by experts as indicated by a greater proportion of fixations between rather than on pieces, and a greater proportion of fixations on salient pieces. A parsimonious explanation of these phenomena is to propose the more efficient encoding of larger chunks by experts (e.g., Reingold, Charness, Pomplun, \& Stampe, 2001). Such skilled encoding leads to rapid recognition of salient relations among distant pieces, thereby enabling the player to focus on appropriate parts of the board. These processes set the stage for the generation of plausible moves that enable swifter and more accurate problem solving.

The analysis of the spatial distribution of early fixations (more empty-square fixations by experts) supports the conclusion that superior domain-specific knowledge allows expert chess players to perceptually encode chess configurations, rather than individual pieces. The finding that piece saliency influences the selection of experts' saccadic endpoints during the first 1-2 sec following display onset clearly supports the role of parafoveal or peripheral processing of chess configurations in guiding their eye movements and is consistent with the view that skilled players can encode chess-relation information in parallel (Reingold, Charness, Schultetus, \& Stampe, 2001). This is the case because random or systematic region-by-region scanning patterns (e.g., a reading-like pattern from the top-left to the bottom-right section of the chessboard) would not be expected to result in similar findings of saccadic selectivity by piece salience.

Thus, the present findings are consistent with the suggestion of Chase and Simon (1973a, 1973b), Gobet and Simon (1998), and de Groot (1946/1978) that a perceptual advantage is a fundamental component of chess skill. Our results are also consistent with other demonstrations of superior perceptual encoding of chess-related material by experts in immediate recall tasks (e.g., Chase \& Simon, 1973a, 1973b; de Groot 1946/1978; de Groot \& Gobet, 1996; Gobet \& Simon, 1996a, 1996b; see Gobet, 1998, for a review), check-detection tasks (Church \& Church, 1983; Milojkovic, 1982; Saariluoma, 1984), enumeration tasks (e.g., count the number of bishops, Saariluoma, 1985, 1990), and a same-different task for side-by-side quarterboard positions (Ellis, 1973).

An important contribution of this study is the generalization of skill-related rapid extraction of chess relations to the "gold standard" move-choice task. Prior tasks, 
Position A: Intermediates

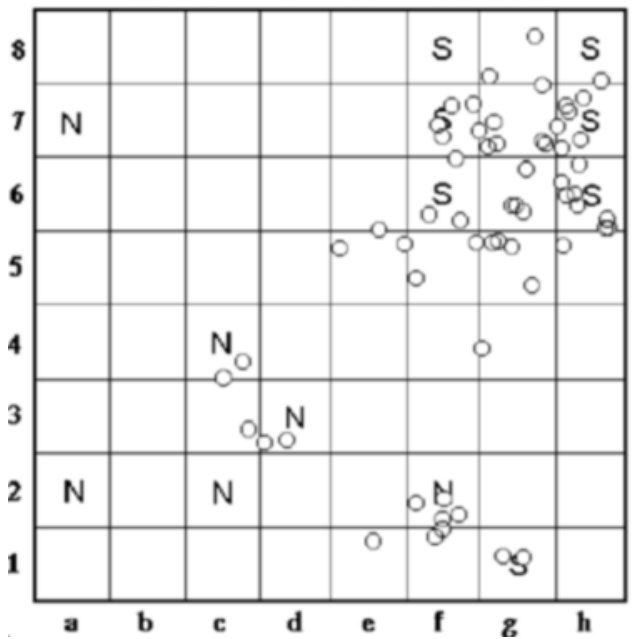

Position A: Experts

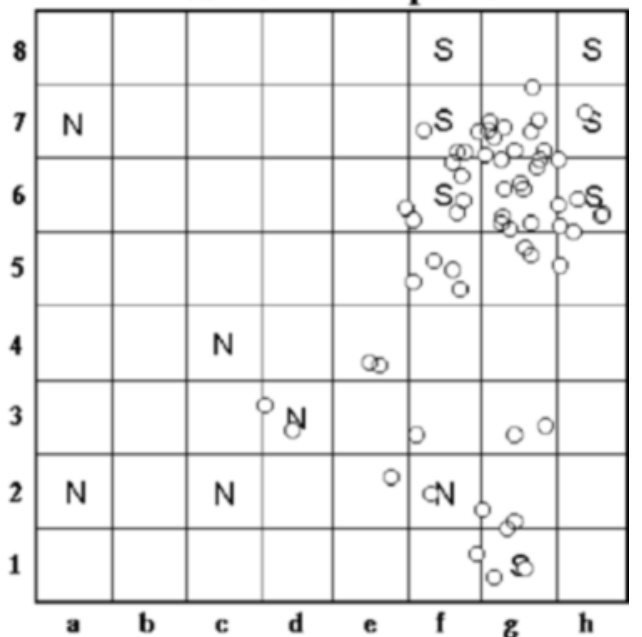

Figure 3. Scattergrams of gaze positions corresponding to the first five fixations produced by intermediates (left panel) and experts (right panel) while attempting to choose the best move in Position A (see Figure 1). $S$ = salient piece; $N=$ nonsalient piece.

such as partial chessboard check detection, full chessboard memorization, and gaze-contingent change blindness, lack the ecological validity of the move-choice task. Nonetheless, the pattern of results revealed by eye-movement data has proven to be quite consistent across both artificial and natural chess tasks. Still, theory development would benefit from future studies that require the same chess players to perform multiple chess-related tasks; it would then be feasible to examine intertask relationships.

De Groot (1946/1978) argued that one of the keys to skill in chess lies not in the thought processes that constitute search through the tree of move possibilities, but rather in the initial encoding of the relationships among the pieces in a chess position. As he put it: "It is not easy to appreciate fully the enormous effect of the expert's reproductive completion of the perceived situation, as his perceptual advantage might be called" (p. 307). Although later research using think-aloud procedures has shown some skill-related depth of search differences between intermediate and expert players (Charness, 1981), numerous studies have supported the importance of initial encoding processes. Eye-movement recording techniques allowed us to track this encoding advantage back to the first second of viewing time as players attempted to choose the best move in a chess game.

\section{REFERENCES}

Chapman, P. R., \& Underwood, G. (1998). Visual search of driving situations: Danger and experience. Perception, 27, 951-964.

Charness, N. (1981). Search in chess: Age and skill differences. Journal of Experimental Psychology: Human Perception \& Performance, 7, 467-476.

Chase, W. G., \& Simon, H. A. (1973a). The mind's eye in chess. In W. G. Chase (Ed.), Visual information processing (pp. 215-281). New York: Academic Press.

Chase, W. G., \& Simon, H. A. (1973b). Perception in chess. Cognitive Psychology, 4, 55-81.
Church, R. M., \& Church, K. W. (1983). Plans, goals, and search strategies for the selection of a move in chess. In P. W. Frey (Ed.), Chess skill in man and machine (2nd ed., pp. 131-156). New York: Springer-Verlag.

De Groot, A. D. (1978). Thought and choice in chess (2nd ed.). The Hague: Mouton. (Original work published 1946)

De Groot, A. D., \& Gobet, F. (1996). Perception and memory in chess. Assen, The Netherlands: Van Gorcum.

ELLIS, S. H. (1973). Structure and experience in the matching and reproduction of chess patterns (Doctoral dissertation, Carnegie-Mellon University, 1973). Dissertation Abstracts International, 73(26), 954.

Goвet, F. (1998). Expert memory: A comparison of four theories. Cognition, 66, 115-152.

Gobet, F., \& Simon, H. A. (1996a). Recall of rapidly presented random chess positions is a function of skill. Psychonomic Bulletin \& Review, 3, 159-163.

Gobet, F., \& Simon, H. A. (1996b). Templates in chess memory: A mechanism for recalling several boards. Cognitive Psychology, 31, $1-40$.

Gobet, F., \& Simon, H. A. (1998). Expert chess memory: Revisiting the chunking hypothesis. Memory, 6, 225-255.

Gobet, F., \& Simon, H. A. (2000). Five seconds or sixty? Presentation time in expert memory. Cognitive Science, 24, 651-682.

Holding, D. H. (1985). The psychology of chess skill. Hillsdale, NJ: Erlbaum.

Horowitz, I. A. (1972). Winning chess tactics. New York: Cornerstone Library.

Jongman, R. W. (1968). Het oog van de meester [The eye of the master]. Assen, The Netherlands: Van Gorcum.

Kundel, H. L., \& Nodine, C. F. (1975). Interpreting chest radiographs without visual search. Radiology, 116, 527-532.

MiloJkovic, J. D. (1982). Chess imagery in novice and master. Journal of Mental Imagery, 6, 125-144.

Newell, A., \& Simon, H. A. (1972). Human problem solving. Englewood Cliffs, NJ: Prentice Hall.

RAYNER, K. (1998). Eye movements in reading and information processing: 20 years of research. Psychological Bulletin, 124, 372-422.

Reinfeld, F. (1945). Win at chess. New York: Dover.

Reingold, E. M., Charness, N., Pomplun, M., \& Stampe, D. M. (2001). Visual span in expert chess players: Evidence from eye movements. Psychological Science, 12, 48-55.

Reingold, E. M., Charness, N., Schultetus, R. S., \& Stampe, D. M. (2001). Perceptual automaticity in expert chess players: Parallel encoding of chess relations. Psychonomic Bulletin \& Review, 8, 504-510. 
REYNOLDS, R. I. (1982). Search heuristics of chess players of differen calibers. American Journal of Psychology, 95, 383-392.

SAARILUOMA, P. (1984). Coding problem spaces in chess (Commentationes Scientiarum Socialium, Vol. 23). Turku, Finland: Societas Scientiarum Fennica.

SAARILUoma, P. (1985). Chess players' intake of task-relevant cues. Memory \& Cognition, 13, 385-391.

SAARILUOMA, P. (1990). Apperception and restructuring in chess players' problem solving. In K. J. Gilhooly, M. T. G. Keane, R. H. Logie, \& G. Erdos (Eds.), Lines of thought: Reflections on the psychology of thinking (pp. 41-57). London: Wiley.

SimON, H. A., \& BARENFELD, M. (1969). Information-processing analysis of perceptual processes in problem solving. Psychological Review, 76, 473-483.
Simon, H. A., \& Chase, W. G. (1973). Skill in chess. American Scientist, 61, 394-403.

Simon, H. A., \& Gilmartin, K. (1973). A simulation of memory for chess positions. Cognitive Psychology, 5, 29-46.

Tikhomirov, O. K., \& PoznyansKaya, E. (1966). An investigation of visual search as a means of analyzing heuristics. Soviet Psychology, 5, 2-15.

WINIKOFF, A. W. (1967). Eye movements as an aid to protocol analysis of problem solving behavior. Unpublished doctoral dissertation, Carnegie-Mellon University, Pittsburgh.

Yarbus, D. L. (1967). Eye movements and vision. New York: Plenum.

(Manuscript received February 5, 2001; revision accepted for publication July 2, 2001.) 